\title{
The auditory tau effect and memory for pitch
}

\author{
IAN P. CHRISTENSEN \\ University of Manchester, Manchester M13 9PL, England \\ and \\ YIH LERH HUANG \\ Carleton University, Ottawa, Ontario KIS 5B6, Canada
}

\begin{abstract}
The tau effect, which designated the effect of temporal variables on spatial perception, was investigated in the auditory modality. Subjects listened to repeated presentations of a three-tone sequence. The first and third tones were fixed in frequency at 1 and $3 \mathrm{kHz}$, respectively, and the subject adjusted the frequency of the second tone until it seemed intermediate in pitch between the other two. The two temporal intervals delimited by the three tones were manipulated. As the second tone moves from being closer to the first tone to being closer to the third tone in time, subjects' average adjustment of the second tone decreases. While in accordance with previous results, the present data provide new information on subjects' behavior in pitch bisection judgments. An explanation for the auditory tau effect is offered in terms of memory for pitch in which the dynamic role of time is taken into account.
\end{abstract}

The tau effect (Helson \& King, 1930) and the kappa effect (Cohen, Hansel, \& Sylvester, 1954; Geldreich, 1934) are usually cited as evidence that the processing of temporal and spatial information is related, at least within some limited set of values of the two stimulus dimensions concerned.

The tau effect refers to situations in which a temporal independent variable influences judgments of a spatial dependent variable. In the terms of Helson's classic demonstration, if three points on a line are marked on the skin of the forearm (delineating two equal distances, $d_{1}$ and $d_{2}$ ) and these points are successively stimulated (thereby creating two interstimulus intervals, $t_{1}$ and $t_{2}$ ), then $d_{1}$ will appear to the subject to be equal to $d_{2}$ only if $t_{1}$ equals $t_{2}$. If $t_{1}>t_{2}, d_{1}$ appears greater than $d_{2}$, and vice versa.

The kappa effect occurs when a spatial independent variable is used, and time becomes the dependent measure. Cohen et al. (1954) used three lights to delineate two distances, $d_{1}$ and $d_{2}$, on a straight line. These lights were made to flash briefly, in succession, giving rise to two interstimulus intervals, $t_{1}$ and $t_{2}$, as before. The subject's task in this case was to adjust the timing of the occurrence of the second light so that it appeared halfway, in time, between the first and third lights. The data from this experiment were analogous to those of Helson. If $\mathrm{d}_{1}=\mathrm{d}_{2}$, then, when the subject has made his adjustments, $t_{1}$ is found (more or less) to equal $t_{2}$. However, if, say, $d_{1}>d_{2}$, then, when the subject has adjusted $t_{1}$ and $t_{2}$ to subjective equality, we find that, in fact, $t_{2}>t_{1}$.

These basic findings have proved to be quite robust, and both the tau and the kappa effects have been shown to occur in the visual, auditory, and tactile modalities with a variety of different stimulus displays and display parameters; Ono (1976) has provided a review of the published data in these and related areas.

It seems not unlikely that some common underlying central mechanism is responsible for these two phenomena, and if that is so we should not be surprised to find other examples of sensory judgments which, in the same manner, are not independent of the timing of the stimuli to which they related. A case in point arises in a certain kind of pitch bisection experiment, in which a subject is required to adjust one tone so that it appears to be halfway in pitch between two others. The three tones are presented in a sequence of fixed length which is repeated until the subject is satisfied with his judgment. If the second tone is, temporally, midway between the first and third tones, then the subject can, in fact, bisect the tonal interval, although individual variances tend to be rather large. However, if the second tone occurs closer, in time, to the first tone than to the third, then the subject will bias his adjustment of the pitch of the second tone towards that of the third, and vice versa (Cohen, Christensen, \& Ono, 1974; Cohen et al., 1954). It had been suggested that this might be an example of the tau effect, the argument being that each tone would be spatially represented on the basilar membrane by a distinct local maximum of excitation, thus making the situation analagous, in a way, to Helson's original points-on-the-skin demonstration. It is now known, of course, that such maxima are by no means as distinct as was originally supposed, and such an 
analogy must be regarded as tenuous to say the least. However, there are distinct similarities between the situations, and the experiment reported here sought to investigate the effects in a more extensive manner than before to see if any further light could be shed on the matter.

\section{METHOD}

\section{Apparatus}

Three sine-wave tones were generated by three Farnell waveform generators. A timing device built within the Department of Psychology at the University of Manchester switched these tones on and off according to the scheme shown in Figure 1; rise and fall times were such as to avoid switching transients.

Previous work has shown that monaural and binaural presentation give equivalent results (Cohen et al., 1974), and so the tones were presented to the subject's right ear only through TDH-39 headphones at $56 \mathrm{~dB}$ SPL. The apparatus was hidden from the subject, who adjusted the pitch of the second tone using the signal generator dial which was behind a cloth screen. The first and third tones were fixed at 1 and $3 \mathrm{kHz}$, respectively; the frequency of the adjustable tone was determined with an SLE 2000 timer/counter at the end of each trial.

\section{Subjects}

Eight subjects were used in this experiment, five male and three female. They were drawn from the student population of the Department of Psychology and were allocated as part of their course requirements. The only constraint applied was that they possess no musical skills, since pilot work had shown that musically trained subjects are very accurate in this task, showing the effect we were investigating only for a few trials while becoming accustomed to the experimental conditions.

\section{Procedure}

In much previous research, the temporal variable has been expressed as the ratio $t_{2} / t_{1}$. As has been noted (Huang, 1976), this is unsatisfactory since it gives rise to a nonlinear scale. In this research, therefore, we adopted the convention of representing the values of the variable as the ratio $t_{1} /\left(t_{1}+t_{2}\right)$.

Nine values of this variable, $.02, .05, .10, .25, .50, .75, .90$, .95 , and .98 , were chosen, and the subjects made 10 judgments under each value, the whole 90 trials being presented in a random order which was the same for each subject. Subjects completed their judgments in three sessions, separated by at least 2 days previous work having shown that long sessions are tiring and lead to boredom.

At the beginning of the first session, the subjects listened to a few repetitions of the cycle with the time variable set at .5 and the second tone at some arbitrary value between 1 and
$3 \mathrm{kHz}$. They were shown how to change the pitch of the second tone and told that they were to adjust it until it seemed to be "halfway in pitch" between the other two.

After the subject was familiarized with the equipment, the timing was set to the first value on the random list, the second tone arbitrarily reset, and the subject instructed to proceed with the task. When the subject was satisfied with his judgment, the frequency of the second tone was recorded and the timing was set to the next value on the list. (Since the potentiometer which controlled the timing emitted a slight noise when operated, it was twiddled before each trial, even when the random list dictated that the same time interval was to occur on consecutive trials.) The frequency of the second tone was then arbitrarily altered by the experimenter, and the whole process repeated. The subjects were not given feedback about their performance.

At the beginning of the second, and subsequent, sessions, the subject listened to the sequence of tones set to the values of the last trial of the preceding session before carrying on with their judgments. It was hoped that this would provide them with some continuity of reference between sessions.

\section{RESULTS}

The data are presented in Table 1 and Figure 2.

Analysis of variance showed a significant effect both of the time variable $(F=5.00, p<.01)$ and subjects $(F=211.10, p<.001)$. Furthermore, the downward linear trend apparent in Figure 2 was found to be significant $(F=416.9, p<.001)$, but the quadratic and cubic trends were not.

In outline, these findings are perfectly in accordance with those reported by Cohen et al. (1974) and Cohen et al. (1954). Both these studies used the same three values of the temporal variable (corresponding, in our notation, to $.33, .50$, and .67), and their results show the same decreasing curvilinear trend evident in the central portion of the graph, although the slopes differ, as do the actual values of the data points. Our data also agree with the earlier findings of one of us (Huang, 1976), with the same reservations. This agreement is, of course, encouraging, but these earlier researches used limited ranges of values of the independent variable and our intention is to provide a more complete picture by a detailed analysis of data covering the whole range.

A histogram of the raw data (Figure 3 ) reveals a distribution which appears to have two distinct

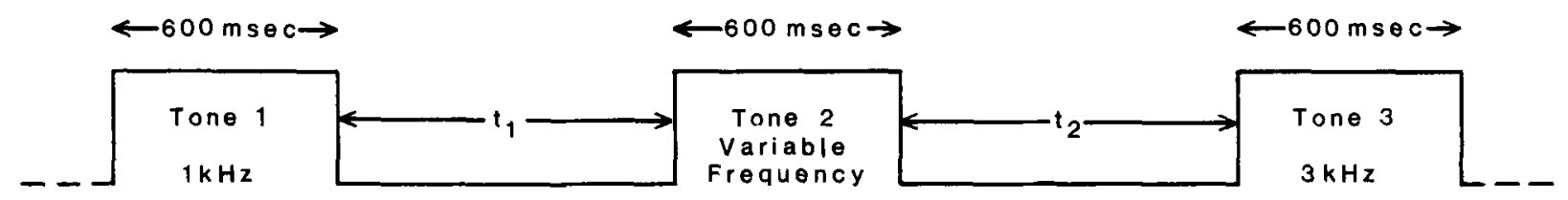

$$
\begin{aligned}
& t_{1}+t_{2}=1.35 \text { sec. } \\
& \text { Duration of Cycte }=3.15 \mathrm{sec}, \\
& \text { Inter-cycle Interval }=3.35 \mathrm{sec} \text {. }
\end{aligned}
$$

Figure 1. The timing of one cycle of the stimulus presentation. 
Table 1

Means (M) and Standard Deviations (SD) of Raw Data

\begin{tabular}{lrrrrrrrrrr}
\hline & \multicolumn{8}{c}{ Value of $t_{1} /\left(t_{1}+t_{2}\right)$} \\
\cline { 2 - 10 } & .02 & .05 & .10 & .25 & .50 & .75 & .90 & .95 & .98 \\
\hline M & 1838 & 1802 & 1762 & 1822 & 1762 & 1756 & 1718 & 1686 & 1762 \\
SD & 455 & 403 & 474 & 427 & 483 & 409 & 473 & 392 & 435 \\
\hline
\end{tabular}

Note $-N=80$ for each value.

peaks, one in the region of $1,500 \mathrm{~Hz}$ and the other in the region of $2,000 \mathrm{~Hz}$. We noted that some subjects made their responses almost exclusively in one region or the other, but others shifted between the two without any obvious pattern. It is possible that we may be dealing with data drawn from a binary mixture of two distributions. Although $n$ is not large enough to apply a formal test (see Falmagne, 1968), a less rigorous technique to investigate the possibility was used. We divided the data at the midpoint between the two peaks $(1,750 \mathrm{~Hz})$ and derived two further graphs (Table 2, Figure 4), which are very similar in form to the plot of the complete set of data, but widely separated from each other. The variances of the data points of these partitioned data, are, of course, considerably reduced.

\section{DISCUSSION}

Before discussing the meaning of the statistically significant effect of the time variable, it is interesting to consider why the data can be partitioned in the way we have described while retaining the principal features of the pooled data. The task we set the subjects is a difficult one, and we might ask how a person with no musical training might, in fact, carry it out. One answer to this question is suggested by the values of the two peaks of the distribution; $1,500 \mathrm{~Hz}$ is 1 octave below the third tone, and $2,000 \mathrm{~Hz}$ is 1 octave above the first tone. Subjects' perceptions of the midpoint might very well be influenced by these relationships, and they may indicate satisfaction with their judgments when a consideration of the interval between either the first and second or the second and third tones seems satisfactory. This finding means that data on pitch bisection in this type of experimental situation should be regarded with caution, because our data at the point where $t_{1} /\left(t_{1}+t_{2}\right)$ $=.5$ (that is to say, where the three tones are equally spaced in time, as they would be in a standard pitch bisection task) have a mean value of $1,762.2 \mathrm{~Hz}$, which is very close to the geometric, or musical, midpoint of the interval $(1,732 \mathrm{~Hz})$. Viewed in isolation, one would conclude that our subjects were, on average, quite accurate. However, if our data is truly drawn from a binary mixture of two distributions, that figure is unreliable, since it is derived from averaging results drawn from one distribution with a mean in the region of $1,500 \mathrm{~Hz}$ and another with a mean of $2,000 \mathrm{~Hz}$. In this particular temporal condition we made 80 observations and only three of the eight subjects made any responses at all in the range of $1,700-1,800 \mathrm{~Hz}$; two made one response each in that area, and the third made four. The effect of averaging in this situation is simply to create an artifact.

We must now consider whether this possible use of octaves might not have given rise to the shape of the function we have plotted, and to do this we must make an assumption about how the subjects, in fact, behaved. The obvious one is that when the second tone was closer in time to the first tone, then the influence of the latter predominated, and when the second tone was closer to the third, then the latter predominated. The effect of this, in simple terms, would be that as $t_{1} /\left(t_{1}+t_{2}\right)$ varied from .02 to .98 ,

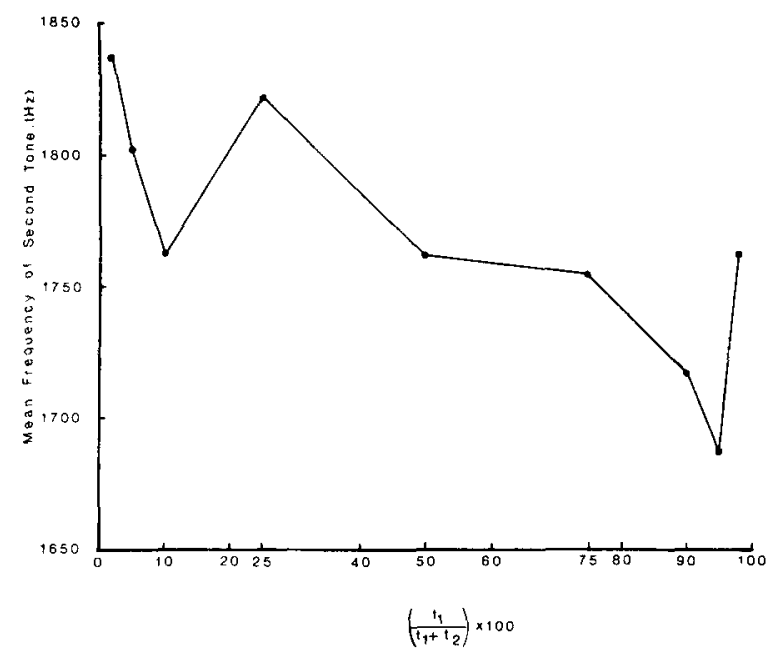

Figure 2. Mean frequency of the second tone as a function of the temporal position of that tone.

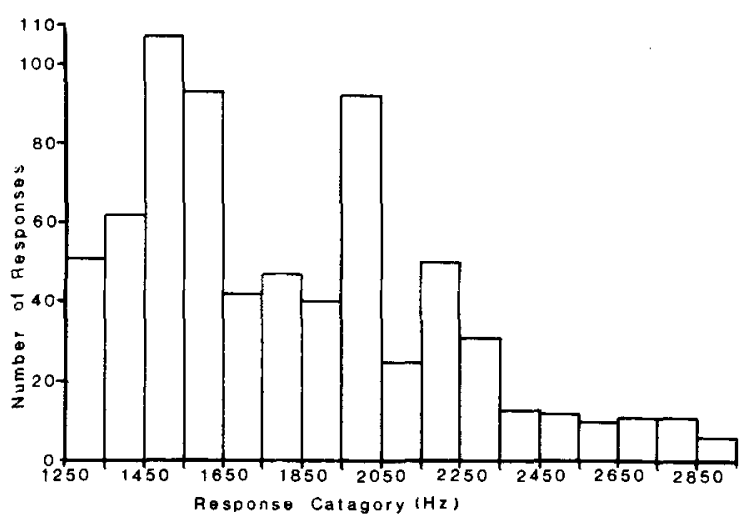

Figure 3. The distribution of subjects' responses. 
Table 2

Partitioned Data

\begin{tabular}{lrrrrrrrrr}
\hline & .02 & .05 & .10 & .25 & .50 & .75 & .90 & .95 & .98 \\
\hline \multicolumn{8}{c}{ Responses } & Under 1,750 & $\mathrm{~Hz}$ \\
$\mathrm{M}$ & 1521 & 1501 & 1508 & 1538 & 1508 & 1511 & 1492 & 1475 & 1464 \\
$\mathrm{SD}$ & 130 & 110 & 102 & 105 & 116 & 140 & 130 & 124 & 135 \\
$\mathrm{~N}$ & 38 & 39 & 36 & 38 & 37 & 43 & 44 & 49 & 41 \\
\multicolumn{8}{c}{ Responses of } & $1.750 \mathrm{~Hz}$ and & Above \\
$\mathrm{M}$ & 2171 & 2064 & 2108 & 2151 & 2099 & 2112 & 2122 & 2085 & 2134 \\
$\mathrm{SD}$ & 305 & 393 & 228 & 268 & 275 & 240 & 250 & 217 & 293 \\
$\mathrm{~N}$ & 42 & 41 & 44 & 42 & 43 & 37 & 36 & 31 & 39 \\
\hline
\end{tabular}

the judgments of our subjects would vary from the region of $2,000 \mathrm{~Hz}$. to the region of $1,500 \mathrm{~Hz}$. A glance at Figure 2 will show that this is apparently what happens, the linear trend being significant. However, this is oversimplifying the matter. In the first place, if our assumption (or, for that matter, its converse) were true, then one would expect the subjects, collectively, to make more responses in the region of $2,000 \mathrm{~Hz}$ when $t_{1}<t_{2}$ and more in the region of $1,500 \mathrm{~Hz}$ when $t_{1}>t_{2}$. This is not the case; Table 2 shows that the number of responses in each region is approximately equal under each temporal condition $\left(\chi^{2}=6.88, \mathrm{df}=8\right.$, n.s.). The fact that the partitioned data show the same trends as the overall data is added evidence to suggest that although our subjects' judgments were not made in the manner that the classical psychophysical literature might lead one to expect, overlying this there remains an effect of the temporal variable which is apparently the tau effect.

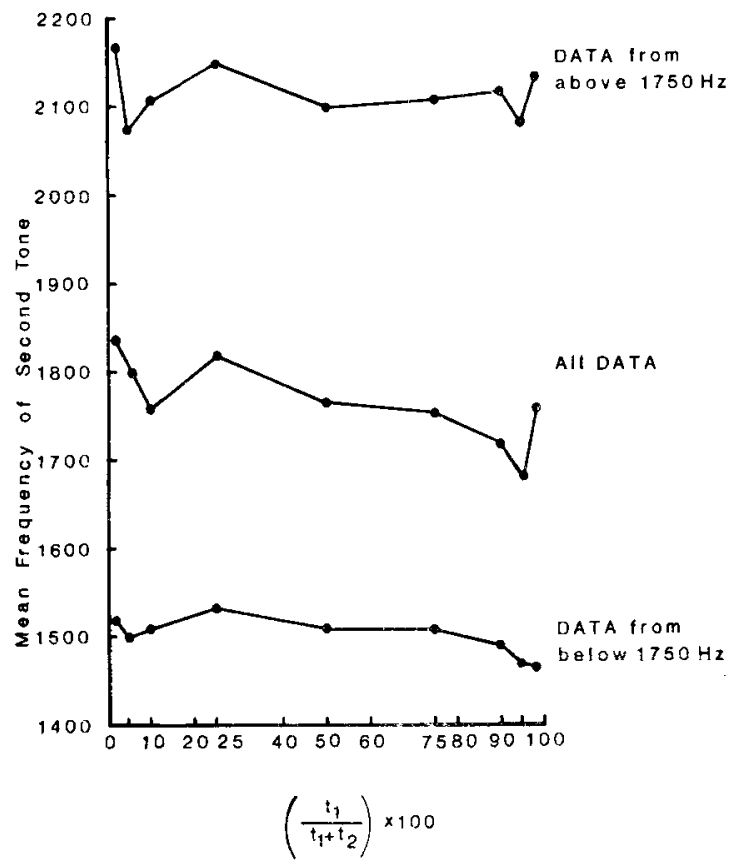

Figure 4. The data partitioned at 1,750 $\mathrm{Hz}$ into two samples.

\section{What is Recalled from Pitch Memory?}

Deutsch (1970a) did an experiment in which subjects heard two brief tones, a standard (S) and a comparison (C), separated by a 6-sec silent interval. C was either the same as $\mathrm{S}$ or one semitone away from it in either direction, and subjects had to respond "same or different" to the two tones. A group of subjects who scored $100 \%$ on this task went on to do an experiment in which their task was the same, but the interval between $S$ and $C$ was no longer silent but was filled with eight notes which they were instructed to ignore. Under these conditions, the subjects' performance deteriorated to nearly chance level. Deutsch (1970b) further showed that this deterioration might be ascribed to the degradation of information in a specialized pitch memory rather than to disturbances of attention, or to interference with information in a general memory store of limited capacity. There is evidence to show that material in this store is capable of consolidation, since if the $\mathrm{S}$ tone is included in the interpolated sequence, recognition performance is enhanced; this effect is more pronounced if the $S$ tone is included earlier rather than later in the sequence (Deutsch, 1975).

Following the initial observation of the degradation of recognition performance when irrelevant tones were interpolated in the retention interval, Deutsch (1972) examined more closely the relationship between performance and the frequency of the interpolated notes. In this experiment, six notes were inserted in the retention interval. Five of these were chosen at random from a range of 24 centered on the $\mathrm{F}$ above middle $\mathrm{C}$, while the sixth, which was always in the second serial position in the sequence, bore a critical relationship to the $S$ tone. On any trial, the $S$ tone itself was chosen at random from the octave middle $\mathrm{C}$ to the $\mathrm{B}$ above. The critical relationship was varied, in intervals of $1 / 6$ tone, from $1 / 6$ to a whole-tone separation. Again, the $C$ tone was either the same as the $\mathrm{S}$ tone or 1 semitone above or below it, and when they differed, the critical tone was placed on the same side of the $\mathrm{S}$ tone along the pitch continuum as was the $\mathrm{C}$ tone. As before, the subjects were simply required to say whether the $\mathrm{S}$ and $\mathrm{C}$ tones were the same or not. The data revealed a systematic relationship between subjects' performance (measured as percent correct) and the tonal separation between the $S$ tone and the critical tone. As the separation increased, there was a smooth rise in error rate to a maximum of about $35 \%$ at a separation of $2 / 3$ tone, falling away to about $12 \%$ at a whole-tone separation.

This finding is of considerable interest to us here, and we may be in a position to extend the discussion, since our data was collected in a rather different manner. A limitation of the "same-different" paradigm is that while it can tell you what the subject could not do, it does not, by a study of mistakes, 
give you any insight into what he was doing when he was wrong. The best kind of model that can be evolved from these data is a kind of "muddy water" system in which the interfering tone is assumed to increase the variance of the trace of the $\mathrm{S}$ tone stored in memory so that when a response is required the subject compares the $C$ tone (which presumably is virtually undegraded) with a sample drawn from the Stone distribution and responds accordingly. The nature of the interference, and of the sample, is unspecified. While some very powerful inferences can be drawn from these models (e.g., Wickelgren, 1969), they are of no use when considering phenomena such as the one reported above, since they predict changes in the variances of observations (and hence in the accuracy of performance in same-different experiments) but not in the mean values of these observations.

Let us consider how a subject might behave in our experiment. On the first presentation, he has to compute the midpoint of the first tone $\left(T_{1}\right)$ and the third tone $\left(\mathrm{T}_{3}\right)$ by means of some algorithm, $\Gamma\left(\mathrm{T}_{3}, \mathrm{~T}_{1}\right)$, which need not be specified but which we shall assume is consistently applied. We shall call this midpoint $\mathrm{T}_{2}{ }^{\prime}$. Since $\mathrm{T}_{2}{ }^{\prime}$ did not arise from direct sensory input (and also because it simplifies the argument), we shall assume for the time being that it is stored in a manner which renders it proof against interference by incoming tones but not, of course, against decay. On the second presentation of the cycle, $T_{2}$ can be compared with $\mathrm{T}_{2}^{\prime}$ as soon as the former is available and the trial is over if they are judged to be the same. If they are not, then $T_{2}$ ' can be "refreshed" by another calculation and the process is repeated, $T_{2}$ on the $n^{\text {th }}$ trial being compared with the $T_{2}{ }^{\prime}$ derived from the $(n-1)^{\text {th }}$ trial.

If the comparison is made in this way, then the variation we observed in our data is due to variation in $T_{2}{ }^{\prime}$ and the systematic main effect reported is due to a systematic variation in $T_{2}{ }^{\prime}$. This, in turn, must be due to the way in which $T_{2}{ }^{\prime}$ is internally derived rather than to a simple degradation of $T_{2}{ }^{\prime}$ in memory, leading to increased variance.

In order to compute $T_{2}{ }^{\prime}, T_{1}$ must be held in memory until $T_{3}$ arrives, by which time $T_{2}$ will have been presented. Deutsch's data indicate that $T_{2}$ will have interfered with $T_{1}$, and our data seem to indicate that the effect of this interference is not to increase the variance of $T_{1}$ (or, at least, not only to increase the variance ot $T_{1}$ ), but to make the retrieved value of $T_{1}$ appear closer in pitch to $T_{2}$ than was physically the case. Thus, $T_{2}{ }^{\prime}$ has a larger value than would otherwise be the case. A more conventional formulation of this would be that whatever the representation of $T_{1}$ in memory, the effect of $T_{2}$ is to skew the distribution of $T_{1}$ towards $T_{2}$. This effect, reasonably enough, diminishes with time and would presumably asymp- tote at some point where we would be able to make some comment on the nature of $\Gamma\left(T_{3}, T_{1}\right)$. One further point adds weight to this argument. If the tonal sequence used in our experiment were reversed so that $T_{1}=3 \mathrm{kHz}$ and $T_{3}=1 \mathrm{kHz}$, then for small values of $t_{1} /\left(t_{1}+t_{2}\right)$ the representation of $T_{1}$ would again be skewed towards $T_{2}$, giving a lower value of $T_{2}{ }^{\prime}$ than might be expected. As $t_{1} /\left(t_{1}+t_{2}\right)$ increased, this effect would diminish and the resulting graph would have a positive slope, the opposite to that obtained in the present situation. The limited data presented in Cohen et al. (1974) indicates that this would indeed be so.

If $\mathrm{T}_{2}{ }^{\prime}$ is stored in the same way as the stimulus tones, then, in the scheme of things we have described, its value would be subject to interference by $T_{1}$ before comparison with $T_{2}$ takes place. Furthermore, this interference would be in the opposite direction to that described above. However, in our experiment, the interval between the end of $T_{3}$ and the onset of $T_{1}$ on the next trial is more than twice that of the maximum delay between $T_{1}$ and $T_{2}$ on the same trial, so that any interference of $T_{1}$ on $T_{2}{ }^{\prime}$ would be small compared to that of $T_{2}$ on $T_{1}$.

Applying the above reasoning to the data from Deutsch (1972) cited earlier, we might conclude that when her subjects were wrong it was because the sample of the $S$ tone which they retrieved from memory to make their comparisons was of the order of 1 semitone removed from its "true" value. This is because, presumably in order to judge as identical two tones which were in fact 1 semitone apart, one of them must have been distorted by roughly this amount. This does not contradict our present findings; the maximum interference we obtained $(1,838 \mathrm{~Hz})$ is almost exactly one semitone above the musical midpoint of our sequence, $1,834 \mathrm{~Hz}$ being the calculated value. Why it should be that, as Deutsch (1972) noted, performance is better when the $\mathrm{S}$ and $\mathrm{C}$ tones are the same compared with when they differ is not accounted for in the above reasoning. It may, of course, be simply due to a response bias on the part of the subjects; Deutsch herself does not appear to attach much importance to the difference.

Deutsch and Faroe (1975) proposed that this interference effect could be accounted for by a lateral inhibition process. The suggestion was based on three pieces of evidence: the shape of the initial error curve (Deutsch, 1972), the range over which the effect operated, and the accumulation of errors when two critical tones were used, one on either side of the S tone on the pitch continuum (Deutsch, 1973). They derived a mathematical model which accurately predicted the shape of a disinhibition function using the initial error curve as an input. It seems that the present data are less likely to be accounted for by such a process if only because the range of frequency dif- 
ferences we used is much larger than that employed by Deutsch. However, we do seem to have observed interference in pitch memory, and whatever processes underlie that phenomenon, it appears that they are the basis of the auditory tau effect.

\section{REFERENCES}

Cohen, J., Hansel, C. E. M., \& Sylvester, J. D. Interdependence of temporal and auditory judgements. Nature, 1954, 174, 641-642.

Cohen, J., Christensen, I. P., \& ONo, A. Influence of temporal intervals on comparative judgments of pitch. Tohoku Psychologica Folia, 1974, 33, 76-87.

DeuTsch, D. The deterioration of pitch information in memory. Unpublished doctoral dissertation, University of California at San Diego, 1970. (a)

DEuTsch, D. Tones and numbers: Specificity of interference in short term memory. Science, 1970, 168, 1604-1605. (b)

Deutsch, D. Mapping of interactions in the pitch memory store. Science, 1972, 175, 1020-1022.

DeuTsch, D. Interference in memory between tones adjacent in the musical scale. Journal of Experimental Psychology, 1973, $100,228-231$.
Deutsch, D. Facilitation by repetition in recognition memory for tonal pitch. Memory \& Cognition. 1975, 3, 263-266.

Deutsch, D., \& Faroe, J. Evidence for lateral inhibition in the pitch memory system. Perception \& Psychophysics, 1975. 17. 320-324.

Fal.magne, J. C. Note on a fixed-point property of binary mixtures. British Journal of Mathematical and Statistical Psychology, $1968,21,131-132$.

Geldreich, E. W. A lecture room demonstrator of the visual tau-effect. American Journal of Psychology, 1934, 46, 483-485.

Helson, H., \& King, S. M. The tau-effect: An example of psychological relativity. Science, 1930, 71, 536-537.

HuANG, Y. L. The auditory tau-effect: A further study. Unpublished BSc dissertation, University of Manchester, 1976.

ONo, A. A study of the literature on the interrelations between subjective time, distance and speed. Tohoku Psychologica Folia, $1976,35,1-11$.

Wickelgren, W. A. Associative strength theory of recognition memory for pitch. Journal of Mathematical Psychology, 1969. 6, 13-61.

(Received for publication March 7, 1979; revision accepted August 17, 1979.) 